\title{
Non-Communicable Chronic Diseases: The Role of Neonatal Characteristics
}

\author{
Maria Elisabetta Baldassarre, ${ }^{1}$ Antonio Di Mauro, ${ }^{1}$ Annarita Cintoli, ${ }^{1}$ Giuseppina Mincarone, ${ }^{1}$ Silvio \\ Tafuri, ${ }^{1}$ and Nicola Laforgia ${ }^{1}$ \\ ${ }^{1}$ Department of Biomedical Science and Human Oncology, University of Bari, “Aldo Moro", Bari, Italy \\ "Corresponding author: Dr. Antonio Di Mauro, Department of Biomedical Science and Human Oncology, Neonatology and Neonatal Intensive Care Unit, University of Bari, \\ “Aldo Moro", P.zza Giulio Cesare, 11, 70125 Bari, Italy. E-mail: dimauroantonio@msn.com
}

Received 2016 October 11; Revised 2017 May 03; Accepted 2017 July 08.

\begin{abstract}
Background: Non-communicable chronic diseases (NCDs) are still the major cause of mortality and morbidity in young adults. Objectives: The aim of the study was to evaluate the influence of neonatal characteristics on NCD development. Methods: A questionnaire was assessed to evaluate the influence of cesarean section (CS), breastfeeding and prematurity on development of some NCDs. The questionnaire was distributed through an online form.

Results: We received 6,379 responses. According to data, CS was not associated with the development of asthma and allergies, celiac disease, type I and II diabetes or obesity and a tendency to be overweight. The study revealed an inverse association between breastfeeding and risk of asthma and allergies, type II diabetes, obesity and a tendency to be overweight. No association was found in relation to type I diabetes or celiac disease. Preterm birth was not associated with development of asthma and allergy, celiac disease or type I and II diabetes, but seems to have a protective role in the development of obesity.

Conclusions: Many neonatal characteristics could influence the development of NCDs during life. These characteristics could have a direct or indirect influence on neonatal gut establishment with subsequent health implications later in life. Further longitudinal studies are needed to confirm our conclusions.
\end{abstract}

Keywords: Prematurity, Breastfeeding, Cesarean section, Non-communicable Chronic Diseases

\section{Background}

According to the world health organization, NCDs are globally considered a leading cause of mortality and morbidity in young adults (1).

It is well known that NCDs have a multi-factorial pathogenesis in which several factors act together for their development.

Risk factors are more frequent and simpler to detect compared to NCDs, and their control is less expensive than treatment of full-expressed NCDs. Therefore, risk factor detection for NCD prevention has become popular practice in public health.

Socio-economic factors, demographic factors and modifiable lifestyle factors such as physical inactivity, low intake of fruits and vegetables, smoking, and alcohol consumption, have been associated with development of NCDs.

Primary prevention programs, instrumental evolution, pharmacological treatments, and lifestyle changes have been put into place to reduce NCDs mortality and morbidity but the burden of such diseases is still unacceptable.
Although behavioral and biological factors of adult life are known and clearly identified, there is limited evidence with regards to the possible risk factors of the perinatal period.

In the last two decades, physicians have investigated the early phases of life as the moment from which NCDs pathogenesis factors could be outlined and, maybe, forced to reduce their development (2).

Growing evidence supports that early life events play a pivotal role in the programming of NCDs as well as differences in intestinal bacterial colonization. For example, different types of delivery, feeding practice or early antibiotic supplementation, may be responsible for the higher risk of NCD development later in life (3).

\section{Objectives}

This article aimed to investigate the prevalence of selfreported NCDs and their perinatal association among a young adult representative sample. 


\section{Methods}

Data source: an internet-based questionnaire, consisting of 32 questions, was designed using Google Forms (4) and was distributed, as previously described in other studies (5), through our Neonatal Unit Facebook page which is linked to the School of Medicine of the University of Bari “Aldo Moro" Network (6).

The questionnaire comprised four areas of interest: (1) demographical characteristics, (2) neonatal characteristics, (3) family features, and (4) health-related information (Supplementary File).

Demographical characteristics included gender and age. Neonatal characteristics included gestational age, delivery mode (CS or vaginal birth) and feeding type. Feeding type was separated into exclusive breastfeeding, combined bottle feeding with breastfeeding and exclusive bottle feeding. The period of exclusive breastfeeding was registered. Family features included educational level and selfreported family history of asthma or allergies, celiac disease and diabetes (I and II). Educational level was recorded into no education, completion of primary education, completion of secondary education and completion of higher education. Health-related information, by a self-reported prevalence of NCDs among university students, residents and PhD students, was collected. Respondents were asked to report whether they had any one of the following NCDs: asthma or allergies, celiac disease or diabetes (I or II), or were overweight or obese. Being overweight or obese was assessed based on self-reported height and weight data, used in a body mass indicator (BMI) calculator: Being overweight was classified as having a BMI of $\geq 25$ and being obese was classified as having a BMI of 30 . There is not an excluded inquiry form. To be enrolled all answers were considered as obligatory fields of the questionnaire to be completed.

The questionnaire was tested by our trainees for comprehensibility and clarity before the link was posted on our Facebook Page.

The link was online for 4 weeks from January to February 2015. Responders could answer the questionnaire anonymously and could give their consent on line for the answers to be published.

Statistical analyses: the database was uploaded as an Excel file and data were analyzed by Stata MP11 software. Categorical variables were reported as proportions and a chi-square test compared these proportions. Quantitative variables were described as means \pm standard deviations and the means were compared by a t-Student test or an ANOVA test when appropriate.

Model for multivariate analysis was performed to study the association between having asthma or allergies, being overweight or obese, having celiac disease or diabetes (I or II) - outcomes - and gestational age, delivery mode (cesarean section or vaginal birth), feeding type, family features - determinants; we designed a model for each outcome. Being overweight or obese was also investigated as determinants of NCDs in a multivariate analysis model. We calculated OR with $95 \% \mathrm{CI}$ and we performed a z-score test. For all tests a $\mathrm{P}<0.05$ was set as significant.

\section{Results}

Descriptive characteristics: 6,379 people participated in our survey, 5,106 (80\%) of whom were female. The overall average age was $25.6 \pm 9.9$ with a significant difference in age between males $(22.9 \pm 10.8)$ and females $(26.3 \pm 9.5 ; \mathrm{t}$ $=11.2, \mathrm{P}<0.0001)$. All respondents were from Italy. About three in ten (29.9\%) were born by CS. A higher proportion of respondents (73.55\%) had received breast milk but only $36.7 \%$ received exclusive breastfeeding until 6 months of life. 1,091 (17.1\%) were born prematurely at a mean gestational age of $34.7 \pm 3.4$ weeks. $7.7 \%$ of respondents had a family history of celiac disease, $13.2 \%$ of diabetes (I or II) and $35.6 \%$ of allergies (Table 1 ).

2,667 (41.8\%) respondents reported having asthma or allergies, 239 (3.7\%) celiac disease, 51 (0.8\%) type I diabetes and $101(1.6 \%)$ type II diabetes. 1,318 (20.7\%) respondents reported having a BMI of $>25$ and, notably, 349 (5.5\%) were obese (Table 2).

Multivariate analysis showed that subjects who reported having been exclusively breastfed were less likely to have asthma or allergies ( $\mathrm{OR}=0.49,95 \% \mathrm{CI}$ : $0.30-0.78$; $\mathrm{Z}=-3.37 ; \mathrm{P}<0.0001)$, type II diabetes $(\mathrm{OR}=0.63,95 \% \mathrm{CI}: 0.41$ - 0.95; $\mathrm{z}=-2.3 ; \mathrm{P}=0.021)$, be overweight $(\mathrm{OR}=0.74,95 \% \mathrm{CI}$ : $0.59-0.94 ; \mathrm{z}=-2.46 ; \mathrm{P}=0.014)$ or obese $(\mathrm{OR}=0.695 \% \mathrm{CI}$ : $0.48-0.76 ; z=-4.3 ; P<0.0001)$.

Prematurity decreased the risk of becoming overweight $(\mathrm{OR}=0.69,95 \% \mathrm{CI}$ : $0.51-0.93 ; \mathrm{z}=2-42 ; \mathrm{P}=0.015)$. Similarly, results showed that being born before 37 weeks of gestation had a lower likelihood of becoming obese, compared to being born at full term $(\mathrm{OR}=0.65,95 \% \mathrm{CI}$ : 0.47 - 0.91; $\mathrm{z}=-2.51 ; \mathrm{P}=0.012$ ). No association was found between prematurity and development of asthma or allergies, celiac disease or type I or II diabetes ( $\mathrm{P}>0.05)$.

Also no significant associations were found between cesarian section and all NCDs investigated $(\mathrm{P}>0.05)$.

Being overweight $(\mathrm{BMI} \geq 25)$ was associated with allergies $(\mathrm{OR}=1.57,95 \% \mathrm{CI}: 1.08-2.14 ; \mathrm{z}=13.48 ; \mathrm{P}<0.0001)$ and type II diabetes ( $\mathrm{OR}=1.65,95 \% \mathrm{CI}: 1-2.7 ; \mathrm{z}=2.78 ; \mathrm{P}=0.0005)$.

Family features are related to some of the investigated NCDs. A higher educational level in the mother seemed to be a preventive factor in offspring becoming overweight $(\mathrm{OR}=0.95,95 \% \mathrm{CI}: 0.92-0.99 ; \mathrm{z}=2.49 ; \mathrm{P}=0.0013)$ or obese 
Table 1. Descriptive Characteristics

\begin{tabular}{|c|c|c|c|c|}
\hline Variables & Men $1273(20)$ & Women $5106(80)$ & All 6379 & P Value \\
\hline Age & $22.9 \pm 10.8$ & $26.3 \pm 9.5$ & $25.6 \pm 9.9$ & $<0.0001$ \\
\hline Cesarean Section & $444(34.9)$ & $1464(28.7)$ & $1908(29.9)$ & $<0.001$ \\
\hline Breastfeeding & $1023(80.4)$ & $3665(71.8)$ & $4688(73.5)$ & $<0.0001$ \\
\hline Prematurity & 213 & 878 & $1091(17.1)$ & 0.69 \\
\hline Maternal age at birth & $29.9 \pm 5.2$ & $29 \pm 5.3$ & $29.2 \pm 5.3$ & $<0.001$ \\
\hline Family history of celiac disease & $81(6.4)$ & $410(8)$ & $491(7.7)$ & 0.048 \\
\hline Family history of diabetes & $131(10.3)$ & $710(13.9)$ & $841(13.2)$ & 0.001 \\
\hline Family history of allergy & $427(33.5)$ & $1844(36.1)$ & $2271(35.6)$ & 0.089 \\
\hline Percentage of prematurity & $213(16.7)$ & $878(17.1)$ & & \\
\hline
\end{tabular}

${ }^{\mathrm{a}}$ Values are expressed as mean (SD) or No. (\%).

Table 2. Prevalence of NCDs

\begin{tabular}{|c|c|c|c|c|}
\hline Variables & Men 1273 (20) & Women 5106 (80) & All 6379 & P Value \\
\hline Asthma or allergies & $534(41.9)$ & $2133(41.8)$ & $2667(41.8)$ & 0.91 \\
\hline Coeliac disease & $29(2.3)$ & $210(4.1)$ & $239(3.7)$ & 0.002 \\
\hline Type I Diabetes & $12(0.9)$ & $39(0.7)$ & $51(0.8)$ & 0.52 \\
\hline Type II Diabetes & $18(1.4)$ & $83(1.6)$ & $101(1.58)$ & 0.59 \\
\hline Overweight & $334(26.2)$ & $984(19.3)$ & $1318(20.7)$ & $<0.0001$ \\
\hline Obesity & $60(4.5)$ & $289(5.7)$ & $349(5.5)$ & 0.19 \\
\hline
\end{tabular}

${ }^{\mathrm{a}}$ Values are expressed as No. (\%).

$(\mathrm{OR}=0.92,95 \% \mathrm{CI}: 0.88-0.96 ; \mathrm{z}=-4.1 ; \mathrm{P}<0.0001)$. The same correlation was found for the father's educational level (OR $=0.94,95 \% \mathrm{CI}$ : $0.91-0.98 ; \mathrm{z}=-3.14 ; \mathrm{P}=0.0002$ ).

A family history of allergies seems a risk factor for allergy and asthma development $(\mathrm{OR}=2.43,95 \% \mathrm{CI}$ : 2.11 - 2.79; $\mathrm{Z}=13.48 ; \mathrm{P}<0.00001)$. Family history of celiac disease is a risk factor for celiac disease $(\mathrm{OR}=5.3,95 \% \mathrm{CI}: 2.29-12.42 ; \mathrm{z}=$ $12.24 ; \mathrm{P}<0.0001)$ and type I diabetes $(\mathrm{OR}=2.24,95 \% \mathrm{CI}: 1.05$ $4.81 ; \mathrm{z}=1.97 ; \mathrm{P}=0.048$ ) development. Family history of type I diabetes is a risk factor for celiac disease development (OR $=4.6,95 \% \mathrm{CI}: 1.36-15.8 ; \mathrm{z}=2.46 ; \mathrm{P}=0.014)$.

\section{Discussion}

The aim of this survey was to estimate the prevalence of self-reported NCDs in early adulthood and their association with neonatal risks factors.

In recent decades, there has been an increase in the prevalence of NCDs in westernized countries. Changes in the occurrence of NCDs in immigrating populations' offspring suggests that early life events and shared environ- mental risk factors may be predetermining NCDs in early adulthood.

The rate of CS, premature births and the trend of bottle feeding have also increased in recent decades and we hypothesized these elements as examples of a shared environmental risk factors for NCDs.

The setting around CS and premature births is different from a full term, vaginal birth with respect to several factors such as antibiotics usage and the hospital environment. The early phases of life affect the microbiome composition of newborns and it could be speculated that the shared environmental risk factors, that may be programming NCDs later in life, are mediated by changes in the microbiome of newborns. Furthermore, non-exposure to labor results in persistence of fetal gene expression and an altered metabolism (7). In the same way, breastfeeding exposes the child to a composite microbiome different from the one encountered during bottle-feeding $(8,9)$.

There are several indications that the diversity and composition of the human microbiome is associated with a variety of diseases (10).

To date, studies addressing the possibility of an asso- 
ciation between CS and NCDs have been small and often contradictory. In recent years, some authors demonstrated an association between CS and the development of asthma and allergies (11), celiac disease (12) and type I diabetes mellitus (13) as well as a tendency to being overweight or obese.

In contrast to these studies, we did not find any association between CS and all six NCDs investigated. Recently an observational study from a Danish nationwide database of children also found no significant association between CS and type I diabetes and celiac disease (14). In line with our results, another study from Brazil, evaluating obesity in a cohort of young adults, showed no effect from mode of delivery (15). A recent population-based prospective birth group of healthy full term newborns observed no clear association between mode of delivery and risk of atopic diseases (16).

Concerning breastfeeding, our study strongly suggests that it is protective against the development of being overweight or obese, or developing type II diabetes and asthma. Recent meta-analyses found positive association with breastfeeding and a reduction of these NCDs (17-19). Breast milk provides the right amount of calories and nutrients the infants needed, changes its composition with time and contains bioactive substances that influence the neonatal immune system, reducing the risk of developing chronic illnesses.

Despite a meta-analysis in 2006 analyzing six observational case-control studies and finding an association between the duration of breastfeeding and a reduced risk of developing Celiac disease (17), our data deny this association. Recently, a multicenter, randomized, interventional trial which explored the relationship between age of gluten introduction and risk of celiac disease reached our same conclusions that breastfeeding did not have a significant impact on the risk of developing celiac disease (18) Concerning the development of type I diabetes, only one study demonstrated a weak protective association between exclusive breast-feeding and developing type I diabetes (19).

With regard to premature births, a few contradictory studies investigated the relationship with developing NCDs. A Puerto Rican cohort study demonstrated no significant association between prematurity and asthma in non-atopic children (20). According to our results, a metaanalysis also showed that although prematurity was associated with asthma in children of all ages, this association became insignificant when the analysis was restricted to studies of older participants (21).

To our knowledge, this is the first study to investigate the separate effects of prematurity on celiac disease, with evidence that there is no association between them.

In contrast to our results, a meta-analysis, including a total of 18 studies for type I diabetes and 5 studies for type II diabetes, suggested that preterm birth was a significant and independent risk factor for both type I and type II diabetes (22).

Finally, our data suggested that a premature birth plays a preventive role in becoming overweight or obese. Differing from our results, a meta-analysis of studies, reporting markers of the metabolic syndrome in adults born preterm, found no association between term and preterm population (23).

We are aware of the limitations of the study: firstly, recall biases are likely since a cross-sectional study was performed and the data on neonatal history was collected retrospectively. Secondly, the way subjects were invited may have affected the high prevalence for some NCDs. Spreading and distributing the questionnaire by means of the Internet and social networks may have inadvertently selected those who are more prone to be affected by NCDs, and this may have influenced the results (i.e. celiac disease prevalence of 3.7\% responders). Furthermore, being responders or university students, or residents or $\mathrm{PhD}$ students, this could be considered a population bias (stratum of society).

Therefore, despite research recruitment using Facebook has been recently described as a potential tool to successfully recruit participants to surveys and clinical studies (24), the results of present study will be used taking into account the declared limitations.

\subsection{Conclusions}

Numerous studies have investigated the association between possible perinatal risk factors and NCDs, some of which have reached conflicting conclusions. According to our data, we can conclude that cesarean section is not a risk factor for the development of NCDs per se via the gut microbiota hypothesis. Future studies should analyze the possible mechanisms involved in NCD pathogenesis in the early stages of life. Understanding associated neonatal risk factors for NCDs is a prerequisite to developing early life interventions, which could help to prevent the increasing epidemic burden of these chronic diseases.

\section{Supplementary Material}

Supplementary material(s) is available here.

\section{Acknowledgments}

The authors acknowledge "Studenti per ..." and "La medicina in uno scatto" for questionnaire diffusion and all students of School of Medicine of University of Bari "Aldo Moro" for responses. 


\section{References}

1. World Health Organization . Global status report on noncommunicable diseases. Geneva: WHO; 2014.

2. Gluckman PD, Hanson MA, Cooper C, Thornburg KL. Effect of in utero and early-life conditions on adult health and disease. $N$ Engl J Med. 2008;359(1):61-73. doi: 10.1056/NEJMra0708473. [PubMed: 18596274].

3. Munyaka PM, Khafipour E, Ghia JE. External influence of early childhood establishment of gut microbiota and subsequent health implications. Front Pediatr. 2014;2:109. doi: 10.3389/fped.2014.00109. [PubMed: 25346925]

4. Google . Create a survey using Google Forms. ; 2014.

5. Amon KL, Campbell AJ, Hawke C, Steinbeck K. Facebook as a recruitment tool for adolescent health research: a systematic review. Acad Pediatr. 2014;14(5):439-447 e4. doi:10.1016/j.acap.2014.05.049. [PubMed: 25169155].

6. Facebook. Health 2015. Available from: https://www.facebook.com/ tinpoliclinicobari?fref $=\mathrm{ts}$.

7. Hyde MJ, Mostyn A, Modi N, Kemp PR. The health implications of birth by Caesarean section. Biol Rev Camb Philos Soc. 2012;87(1):229-43. doi: 10.1111/j.1469-185X.2011.00195.x. [PubMed: 21815988].

8. Rautava S. Early microbial contact, the breast milk microbiome and child health. J Dev Orig Health Dis. 2016;7(1):5-14. doi: 10.1017/S2040174415001233. [PubMed: 26051698].

9. Di Mauro A, Neu J, Riezzo G, Raimondi F, Martinelli D, Francavilla R, et al. Gastrointestinal function development and microbiota. Ital J Pediatr. 2013;39:15. doi:10.1186/1824-7288-39-15. [PubMed: 23433508].

10. Collado MC, Rautava S, Isolauri E, Salminen S. Gut microbiota: a source of novel tools to reduce the risk of human disease? Pediatr Res. 2015;77(1-2):182-8. doi: 10.1038/pr.2014.173. [PubMed: 25335085].

11. Thavagnanam S, Fleming J, Bromley A, Shields MD, Cardwell CR. A meta-analysis of the association between Caesarean section and childhood asthma. Clin Exp Allergy. 2008;38(4):629-33. doi: 10.1111/j.1365-2222.2007.02780.x. [PubMed: 18352976].

12. Marild K, Stephansson O, Montgomery S, Murray JA, Ludvigsson JF. Pregnancy outcome and risk of celiac disease in offspring: a nationwide case-control study. Gastroenterology. 2012;142(1):39-45 e3. doi: 10.1053/j.gastro.2011.09.047. [PubMed: 21995948].

13. Cardwell CR, Stene LC, Joner G, Cinek O, Svensson J, Goldacre MJ, et al. Caesarean section is associated with an increased risk of childhoodonset type 1 diabetes mellitus: a meta-analysis of observational studies. Diabetologia. 2008;51(5):726-35. doi: 10.1007/s00125-008-0941-z. [PubMed: 18292986].
14. Sevelsted A, Stokholm J, Bonnelykke K, Bisgaard H. Cesarean section and chronic immune disorders. Pediatrics. 2015;135(1):e92-8. doi: 10.1542/peds.2014-0596. [PubMed: 25452656].

15. Barros FC, Matijasevich A, Hallal PC, Horta BL, Barros AJ, Menezes AB, et al. Cesarean section and risk of obesity in childhood, adolescence, and early adulthood: evidence from 3 Brazilian birth cohorts. Am J Clin Nutr. 2012;95(2):465-70. doi: 10.3945/ajcn.111.026401. [PubMed: 22237058]

16. Bruske I, Pei Z, Thiering E, Flexeder C, Berdel D, von Berg A, et al Caesarean Section has no impact on lung function at the age of 15 years. Pediatr Pulmonol. 2015;50(12):1262-9. doi: 10.1002/ppul.23196. [PubMed: 25847609].

17. Akobeng AK, Ramanan AV, Buchan I, Heller RF. Effect of breast feeding on risk of coeliac disease: a systematic review and metaanalysis of observational studies. Arch Dis Child. 2006;91(1):39-43. doi: 10.1136/adc.2005.082016. [PubMed: 16287899].

18. Lionetti E, Castellaneta S, Francavilla R, Pulvirenti A, Tonutti E, Amarri $\mathrm{S}$, et al. Introduction of gluten, HLA status, and the risk of celiac disease in children. $N$ Engl J Med. 2014;371(14):1295-303. doi: 10.1056/NEJMoa1400697. [PubMed: 25271602].

19. Cardwell CR, Stene LC, Ludvigsson J, Rosenbauer J, Cinek O, Svensson J, et al. Breast-feeding and childhood-onset type 1 diabetes: a pooled analysis of individual participant data from 43 observational studies.Diabetes Care. 2012;35(11):2215-25. doi:10.2337/dc12-0438. [PubMed: 22837371].

20. Rosas-Salazar C, Ramratnam SK, Brehm JM, Han YY, Boutaoui $\mathrm{N}$, Forno E, et al. Prematurity, atopy, and childhood asthma in Puerto Ricans. J Allergy Clin Immunol. 2014;133(2):357-62. doi: 10.1016/j.jaci.2013.09.003. [PubMed: 24139607].

21. Jaakkola JJ, Ahmed P, Ieromnimon A, Goepfert P, Laiou E, Quansah $\mathrm{R}$, et al. Preterm delivery and asthma: a systematic review and meta-analysis. J Allergy Clin Immunol. 2006;118(4):823-30. doi: 10.1016/j.jaci.2006.06.043. [PubMed: 17030233].

22. Li S, Zhang M, Tian H, Liu Z, Yin X, Xi B. Preterm birth and risk of type 1 and type 2 diabetes: systematic review and meta-analysis. Obes Rev. 2014;15(10):804-11. doi: 10.1111/obr.12214. [PubMed: 25073871].

23. Parkinson JR, Hyde MJ, Gale C, Santhakumaran S, Modi N. Preterm birth and the metabolic syndrome in adult life: a systematic review and meta-analysis. Pediatrics. 2013;131(4):e1240-63. doi: 10.1542/peds.2012-2177. [PubMed: 23509172].

24. Pedersen ER, Kurz J. Using Facebook for Health-related Research Study Recruitment and Program Delivery. Curr Opin Psychol. 2016;9:38-43. doi: 10.1016/j.copsyc.2015.09.011. [PubMed: 26726313]. 